\title{
'Test n Treat (TnT)'- Rapid testing and same-day, on-site treatment to reduce rates of chlamydia in sexually active further education college students: study protocol for a cluster randomised feasibility trial
}

Sarah Kerry-Barnard ${ }^{1}$, Charlotte Fleming ${ }^{1}$, Fiona Reid ${ }^{2}$, Rachel Phillips², Vari M. Drennan ${ }^{3}$, Elisabeth J. Adams ${ }^{4}$, Wendy Majewska ${ }^{5}$, Anjella Balendra ${ }^{1}$, Emma Harding-Esch ${ }^{6}$, Emma Cousins ${ }^{6}$, S. Tariq Sadiq ${ }^{6}$ and Pippa Oakeshott ${ }^{1 *}$ (D)

\footnotetext{
Abstract

Background: Sexually active young people attending London further education (FE) colleges have high rates of chlamydia, but screening rates are low. We will conduct a cluster randomised feasibility trial of frequent, rapid, onsite chlamydia testing and same-day treatment (Test and Treat $(T n T)$ ) in six FE colleges (with parallel qualitative and economic assessments) to assess the feasibility of conducting a future trial to investigate if $\mathrm{TnT}$ reduces chlamydia rates.

Methods: We will recruit 80 sexually active students aged 16-24 years from public areas at each of six colleges. All participants (total $n=480$ ) will be asked to provide samples (urine for males, self-taken vaginal swabs for females) and complete questionnaires on sexual lifestyle and healthcare use at baseline and after 7 months. Participants will be informed that baseline samples will not be tested for 7 months and be advised to get screened separately. Colleges will be randomly allocated to the intervention (TnT) or the control group (no TnT).

One and 4 months after recruitment, participants at each intervention college $(n=3)$ will be texted and invited for on-site chlamydia tests using the 90-min Cepheid GeneXpert system. Students with positive results will be asked to see a visiting nurse health adviser for same-day treatment and partner notification, (backed by genitourinary medicine follow-up). Participants in control colleges $(n=3)$ will receive 'thank you' texts 1 and 4 months after recruitment.

Seven months after recruitment, participants from both groups will be invited to complete questionnaires and provide samples for TnT. All samples will be tested, and same-day treatment offered to students with positive results.

Acceptability of TnT will be assessed by qualitative interviews of purposively sampled students $(n=30)$ and college staff $(n=12)$. We will collect data on costs of TnT and usual healthcare.

(Continued on next page)
}

\footnotetext{
* Correspondence: oakeshot@sgul.ac.uk

'Population Health Research Institute, St George's, University of London,

London SW17ORE, UK

Full list of author information is available at the end of the article
}

(c) The Author(s). 2018 Open Access This article is distributed under the terms of the Creative Commons Attribution 4.0 International License (http://creativecommons.org/licenses/by/4.0/), which permits unrestricted use, distribution, and reproduction in any medium, provided you give appropriate credit to the original author(s) and the source, provide a link to the Creative Commons license, and indicate if changes were made. The Creative Commons Public Domain Dedication waiver (http://creativecommons.org/publicdomain/zero/1.0/) applies to the data made available in this article, unless otherwise stated. 
(Continued from previous page)

Discussion: Findings will provide key values to inform feasibility, sample size and timescales of a future definitive trial of TnT in FE colleges, including:

- Recruitment rates

- TnT uptake rates

- Follow-up rates

- Prevalence of chlamydia in participants at baseline and 7 months

- Acceptability of TnT to students and college staff

- Estimate of the cost per person screened/treated in TnT versus usual care

Trial registration: International Standard Randomised Controlled Trials Registry, ID: ISRCTN58038795, Registered on 31 August 2016.

Keywords: Rapid chlamydia tests, Screening, Young people, Further education colleges, Test and treat, Cluster randomised, Feasibility trial

\section{Background}

There are high rates of sexually transmitted infections (STIs) in ethnically diverse, sexually active students aged 16-24 years attending London further education (FE) colleges [1-4], with around $8 \%$ testing positive for Chlamydia trachomatis. However, uptake of chlamydia screening remains low: below 30\% annually in 16-24-year-olds in England $[4,5]$. Although chlamydia primarily affects young people, the consequences of infection such as infertility, chronic pelvic pain or epididymitis can last a lifetime. It is estimated that $10-16 \%$ of women with untreated chlamydia will develop clinical pelvic inflammatory disease of whom $8 \%$ will have an ectopic pregnancy and $11 \%$ will suffer from tubal-factor infertility [6]. The cost of chlamydia to the NHS is estimated to be over $£ 100$ million each year.

Barriers to reducing chlamydia rates include low uptake of testing by those most at risk $[5,7]$ (such as sexually active teenagers, people from ethnic minorities and people who are socioeconomically deprived), and long delays in receiving a positive diagnosis or attending for treatment. Introducing rapid, on-the-spot chlamydia tests and treatment into the community could make it easier for young people to get tested and treated faster, and before they can pass on their infection. It might also prevent complications $[8,9]$. These novel tests can have 99\% sensitivity and $99.4 \%$ specificity [10], and studies have demonstrated their feasibility in remote communities [11]. However, there have been no UK trials of rapid STI tests and same-day, on-site treatment (Test and Treat $(\mathrm{TnT}))$ in non-healthcare settings.

We will use a test that checks for gonorrhoea as well as chlamydia as this would likely be included in a real-life roll out. However, as people diagnosed with gonorrhoea are best managed by a sexual health clinic they will not receive on-site treatment. Hence, TnT will be just for chlamydia.

Our cluster randomised feasibility trial aims:
1. To assess the feasibility of conducting a trial of TnT in FE colleges, and obtain estimates of key values to inform sample size estimates and timescales for a definitive trial

2. To explore the acceptability of TnT through a qualitative evaluation

3. To estimate the cost per person tested and treated in TnT versus usual care

\section{Design}

Cluster randomised controlled feasibility trial over 7 months with parallel qualitative and economic assessments (Fig. 1 and Additional file 1: Standard Protocol Items: Recommendations for Interventional Trials (SPIRIT) 2013 Checklist (Fig. 2)). The outcome is within one academic year to optimise follow-up.

\section{Setting}

Six ethnically diverse FE colleges in London FE colleges take students from the age of 16 years and teach both academic subjects and vocational courses such as plumbing and hairdressing. As previously [3] we will first obtain agreement from staff and student bodies.

\section{Participants}

At each site during 2 days, 80 consecutive sexually active students (total 480 students across all sites) aged 1624 years will be recruited. We previously found that such students are a high-risk group: $43 \%$ report two or more sexual partners in the past year, 34\% smoke cigarettes, $50 \%$ are teenagers and 30\% are from black ethnic minority groups [3].

\section{Exclusion criteria}

Students who have never had penetrative sexual intercourse; students with severe learning disability. 


\section{Design of Test $\mathbf{n}$ Treat (TnT) cluster randomised feasibility trial}

\begin{tabular}{|c|c|c|}
\hline \multirow{2}{*}{$\begin{array}{l}\text { Recruitment of students } \\
\text { over } 3 \text { weeks followed } \\
\text { by Randomisation of } \\
\text { colleges in } \\
\text { week } 4\end{array}$} & \multicolumn{2}{|c|}{$\begin{array}{l}6 \text { FE colleges agree to take part. } \\
80 \text { sexually active students aged } 16-24 \text { recruited from each } \\
\text { college. Questionnaires, and samples for storage. }\end{array}$} \\
\hline & $\begin{array}{l}3 \text { colleges randomly } \\
\text { allocated to intervention } \\
\text { group ( } \mathrm{TnT})\end{array}$ & $\begin{array}{l}3 \text { colleges randomly } \\
\text { allocated to control group } \\
\text { (no TnT) }\end{array}$ \\
\hline \multirow{2}{*}{$\begin{array}{l}\text { Intervention at } 1 \text { and } 4 \\
\text { months } \\
\text { Parallel qualitative } \\
\text { evaluation exploring } \\
\text { acceptability of TnT }\end{array}$} & $\begin{array}{l}1 \text { month: Texts inviting } \\
\text { participants to TnT. } \\
\text { Rapid tests and treatment }\end{array}$ & $\begin{array}{l}1 \text { month: Texts thanking } \\
\text { participants for being in } \\
\text { study }\end{array}$ \\
\hline & $\begin{array}{l}4 \text { months: Texts inviting } \\
\text { participants to TnT. } \\
\text { Rapid tests and treatment }\end{array}$ & $\begin{array}{l}4 \text { months: Texts thanking } \\
\text { participants for being in } \\
\text { study }\end{array}$ \\
\hline $\begin{array}{l}\text { Outcome assessment at } \\
7 \text { months }\end{array}$ & $\begin{array}{l}\text { Repeat final samples: test, } \\
\text { and treat if necessary } \\
\text { Questionnaire including } \\
\text { data on healthcare use for } \\
\text { cost analysis }\end{array}$ & $\begin{array}{l}\text { Repeat final samples: test, } \\
\text { and treat if necessary } \\
\text { Questionnaire including } \\
\text { data on healthcare use for } \\
\text { cost analysis }\end{array}$ \\
\hline
\end{tabular}

Fig. 1 Shows the design of the Test $\mathrm{n}$ Treat (TnT) cluster randomised feasibility trial

\begin{tabular}{|c|c|c|c|c|c|}
\hline \multirow[b]{3}{*}{ TIMEPOINT $^{\star *}$} & \multicolumn{5}{|c|}{ STUDY PERIOD } \\
\hline & \multirow{2}{*}{$\begin{array}{c}\text { Enrolment } \\
-t_{1}\end{array}$} & \multirow{2}{*}{$\begin{array}{c}\text { Allocation } \\
0\end{array}$} & \multicolumn{2}{|c|}{ Post-allocation } & \multirow{2}{*}{$\begin{array}{c}\begin{array}{c}\text { Follow up } \\
\text { at } 7 \\
\text { months }\end{array} \\
t_{x}\end{array}$} \\
\hline & & & Month $1 \mathrm{TnT}$ & Month $4 \mathrm{TnT}$ & \\
\hline \multicolumn{6}{|l|}{ ENROLMENT: } \\
\hline \multirow{2}{*}{$\begin{array}{l}\text { Eligibility screen } \\
\text { Informed consent }\end{array}$} & $X$ & & & & \\
\hline & $X$ & & & & \\
\hline Allocation & & $\mathrm{X}$ & & & \\
\hline \multicolumn{6}{|l|}{ INTERVENTIONS: } \\
\hline \multicolumn{6}{|l|}{$\begin{array}{c}\text { [TnT test } n \text { treat } @ \\
1 \text { and } 4 \text { months] } \\
\end{array}$} \\
\hline \multicolumn{6}{|l|}{ ASSESSMENTS: } \\
\hline $\begin{array}{r}\text { Provide } \\
\text { genitourinary } \\
\text { samples }\end{array}$ & $x$ & & & & $\mathrm{x}$ \\
\hline Questionnaire & $x$ & & & & $X$ \\
\hline
\end{tabular}

${ }^{*}$ Recommended content can be displayed using various schematic formats. See SPIRIT 2013

Explanation and Elaboration for examples from protocols.

**List specific timepoints in this row.

Fig. 2 Standard Protocol Items: Recommendations for Interventional Trials (SPIRIT) Figure showing the schedule of enrolment, interventions, and assessments for the Test $\mathrm{n}$ Treat $(\mathrm{TnT})$ chlamydia screening feasibility trial 
Recruitment and consent (September to October 2016)

Research assistants will approach students in common room areas $[3,12]$. The students will be asked if they are willing to help with research on sexual health. Students aged 16-24 will be invited to come to the study table where recruiters will explain that as the study is about chlamydia and sexually transmitted infections, only students who have had penetrative sexual intercourse should consider taking part. Those who are interested will be given a patient information sheet and consent form to read and be encouraged to ask questions.

The information sheet will explain that participants will be asked to complete short confidential electronic questionnaires on sexual health using a tablet computer, and to provide samples on this day and again after 7 months (urine for males and self-taken vaginal swabs for females). In half the colleges, students will also be asked to provide samples for rapid testing for chlamydia and gonorrhoea after 1 and 4 months, and those found to be infected with chlamydia will be given same-day, on-site treatment. Some students may also be invited to take part in interviews [13].

Research assistants will ensure that all participants understand that samples provided at recruitment will not be tested for 7 months and it is the student's responsibility to get tested separately [3] (e.g. at a sexual health clinic or at a general practice) if they are allocated to the control group or if they want to be tested for other STIs such as HIV.

These stored baseline samples are for three reasons:

1. To emulate the processes of a future definitive trial

2. To ensure that all participants know how simple it is to provide samples for testing

3. To measure baseline chlamydia prevalence at each site in order to refine the intra-class correlation coefficient (ICC) for sample size calculations

Students who agree to take part will be asked to sign a consent form and to provide full contact details including mobile number and email for follow-up and text reminders [3]. They will be asked for consent to obtain their NHS numbers and to allow the researchers to access their GP, hospital, and genitourinary medicine (GUM) clinic records [14].

\section{Honoraria for participants}

Honoraria will be provided both to facilitate recruitment $[15,16]$ and to encourage participants to return repeat samples at the end of the trial. (We have ethical approval for honoraria and have shown them to be very effective [16]). Participants will be given $£ 5$ when they return completed sample packs at recruitment, and $£ 10$ at the final 7-month follow-up. Participants in the qualitative interviews will be given $£ 10$ for their time. However, participants in intervention colleges will not be given honoraria to attend for TnT after 1 and 4 months as this would not happen were the intervention to be rolled out in future.

\section{Data collection at baseline (September to October 2016)}

During recruitment, participants will be asked to complete a confidential baseline questionnaire using a tablet computer. Questions will include date of birth, self-assigned ethnicity, smoking, alcohol, age at sexual debut, condom use, contraception, number of sexual partners in the previous 12 months, when they last had sex with a new partner, recent STI testing and treatment, history of STI, and genitourinary symptoms in the past 6 months [3]. All participants will also be asked to provide samples in the nearest toilet.

\section{Randomisation}

Randomisation will take place once recruitment is completed and baseline data collected for all colleges.

Using a computer programme, colleges will be randomly allocated into the intervention (TnT) or the control (no TnT) by the trial statisticians RP and FR. The randomisation will be constrained to ensure that three colleges are allocated to each group.

\section{Intervention TnT colleges - rapid chlamydia/gonorrhoea testing and same-day chlamydia treatment ( 1 and 4 months after recruitment)}

In November 2016, 1 month after recruitment, each intervention campus will be visited on two consecutive days by the TnT team. These will be the same days of the week as at recruitment to optimise student attendance. The 80 participating students in each campus will be texted and invited to come for on-site rapid chlamydia/gonorrhoea testing and same-day treatment for chlamydia. As at recruitment they will be invited to complete a questionnaire and provide a sample, but this time the sample will be tested immediately on site using the Cepheid GeneXpert system which takes 90 min. Participants will be given a card containing information about the local GUM/sexual health clinic, a link to the Brook sexual health website: https://www.brook.org.uk, and TnT study contact details.

Negative results will be texted. Participants with positive results will be telephoned by the nurse health adviser and invited to see her in the college nurse's room for confidential same-day treatment if positive for chlamydia, partner notification, advice, and follow-up. Infected students will be asked to bring any sexual partners who attend the college so they can also be tested and treated. (In a survey in 2014, 9\% of 103 students said they had a sexual partner at the same FE college.) Students who are positive for gonorrhoea will be 
asked to attend St George's NHS Trust GUM clinic for further testing and review by a clinician.

All participants in the three intervention colleges will be invited to provide repeat samples for on-site TnT 4 months after recruitment (i.e. the next term JanuaryFebruary 2017).

\section{Control 'usual care' colleges ( 1 and 4 months after recruitment)}

Participants from the three control colleges will not get TnT but will receive texts 1 and 4 months after recruitment thanking them for being in the study.

\section{Data collection at 7-month follow-up (April-May 2017)}

All participants will be asked to provide samples for TnT and to complete questionnaires at college at 7 months. Follow-up questionnaires will include additional questions about STI testing and treatment, oral sex, vaccination against human papillomavirus (HPV), and use of healthcare services for sexual health since recruitment date, including attendances at general practice, sexual health or hospital clinics, hospital admissions, and drug treatment $[3,17]$.

When we invite them to the outcome assessment we will send participants a link to an additional consent form and information sheet explaining that we will be asking them to provide optional mouthwash samples (for future testing for HPV and chlamydia/gonorrhoea) as well as genitourinary samples. We will also put this information on the study webpage. For those who attend we will provide paper copies of the additional consent form and information sheet and answer any questions. We will explain that providing the mouthwash sample is optional and for research purposes only and, that as chlamydia/gonorrhoea and HPV tests are not validated on these samples, we will not feedback results. Those who agree will be asked to sign the additional consent form.

Testing at 7 months is required in the proposed full trial in order to calculate the main outcome (prevalence of chlamydia), and is included here to test the feasibility of collecting these data. It will also help inform estimates of effect size for sample size calculations. Treatment of those diagnosed with infection at 7 months will also be offered. This is not part of the assessed intervention, but is offered to enhance testing uptake at this time, and participation in general among the control group. In addition, at the end of the study stored baseline samples will be tested using standard tests, and students with positive results will be contacted by the health adviser [3].

\section{Masking}

Recruitment of colleges and participants will be conducted prior to group allocation ${ }^{3,19}$. Therefore, the baseline data collection from students will be blind to treatment group. By the time of the first TnT intervention 1 month after baseline, participants and research assistants will no longer be blinded.

\section{Qualitative evaluation exploring the acceptability of TnT}

A research assistant will conduct semi-structured interviews [13] with a purposive sample of male and female college staff and students to investigate views on the acceptability of TnT including barriers and facilitators to uptake and possible harms.

\section{College staff $(n=12)$}

We will interview teaching and student welfare staff and explore opinions of the acceptability of the intervention, barriers, facilitators, harms, and challenges to reaching certain subgroups such as male teenagers from ethnic minorities. We will also explore views on the trial methodology and suggested improvements.

\section{Students $(n=30)$}

These interviews will explore opinions on acceptability, barriers, and facilitators to uptake of $\mathrm{TnT}$, and views of potential harms of on-site rapid tests and treatment. We will also seek views as to the trial methodology and suggested improvements.

Interviews will focus on three distinct groups:

1. Students who declined to participate in the trial $(n=10)$ who agree to be interviewed to explore factors influencing their decision

2. Participants $(n=10)$ in intervention sites who used TnT

3. Participants $(n=10)$ in intervention sites who were recruited but did not use $\mathrm{TnT}$

Interviews will be digitally recorded with permission, transcribed and thematically analysed.

\section{Health economic analysis}

We will estimate the cost per person screened and treated of implementing $\mathrm{TnT}$ in FE colleges compared with usual care (no TnT), and the incremental cost per chlamydia infection averted. Costs will be classified as solely research; capital set-up costs; or on-going running costs. We will also assess the feasibility of obtaining questionnaire data on healthcare use during the study period. Based on what participants report in their 7-month questionnaires about healthcare setting attended for chlamydia-related problems, we will explore the feasibility of estimating the health resources used in both arms of the study using published tariff costs for GUM, hospital inpatient and outpatient visits, and GP attendance [17]. We will explore the marginal costs of offering TnT at 1 and 4 months compared to no test. 
Results will inform the cost of implementing $\mathrm{TnT}$ in a definitive trial.

\section{Main outcomes}

1. Key values to inform feasibility, sample size, and timescales of a full trial of TnT in FE colleges:

(a) Recruitment rates:

- Recruitment rate: colleges and students

- Time taken to recruit 80 participants at each site

- Age, gender, and ethnicity of students recruited versus not recruited [16]

(b) Testing and treatment uptake rates

( 1 and 4 months after recruitment):

- Testing and treatment uptake rates, in intervention sites only

- Time from provision of sample to treatment of chlamydia positives

(c) Follow-up rates (at 7 months):

- Percentage providing samples at all sites

- Percentage completing final questionnaires (including data on healthcare usage)

(d) Prevalence of chlamydia in participants at each site at baseline and at 7 months. Chlamydia prevalence at baseline will enable us to refine the ICC, and prevalence at 7 months to refine the estimated effect size, both required for sample size calculations for the substantive trial

2. A perspective on the acceptability of TnT in FE colleges, emerging from qualitative interviews with purposively sampled students and college staff

3. Health economic analysis (as described above)

Estimate of the cost per person screened/treated in TnT versus usual care.

\section{Sample size and statistical analysis}

Assuming a 30\% recruitment rate [12], 1600 students will be approached to recruit 480 overall ( 80 per site across six sites, three intervention, and three control). Estimates of testing uptake at 1 and 4 months (intervention sites only) will be based on 240 students, and at 7 months will be based on 480 students (all sites).

Teare et al. [18] recommend that 60 to 100 subjects is sufficient to estimate an event rate with acceptable precision in a feasibility study. Prevalence of chlamydia at baseline will be estimated separately for each of the six sites ( 80 students per site), and these prevalences will be used to inform the intraclass correlation coefficient (ICC), required for the sample size calculation for the main study. From our previous research involving 11 colleges [3], the ICC was estimated to be 0.005 (95\% confidence interval (CI) -0.013 to 0.026 ). Adding data from another six colleges will improve the precision of this ICC, reducing the width of the confidence interval by around $20 \%$.

Assuming 70\% followed up at 7 months (with $£ 10$ honoraria), final estimates of chlamydia prevalence would be based on 168 students in each of the intervention and control groups. The study is not powered to find a statistically significant difference, but this may provide useful information on possible effect size to inform future sample size calculations.

\section{Statistical analysis \\ Summary of baseline data and flow of patients}

A Consolidated Standards of Reporting Trials (CONSORT) flow diagram will be produced to show the number of students recruited, the numbers attending at 7-month final follow-up, and for those not attending the reasons (where available) for non-attendance including lost-to-follow-up and withdrawal. Baseline descriptions of students recruited to the study will be presented by treatment arm: including means and standard deviation or numbers and proportions as appropriate. This will be repeated to compare the baseline characteristics of those in the intervention arm returning for TnT at 1 and 4 months compared to those who did not return. Where available reasons for non-attendance at the 1- and 4-month visits will be summarised. Summary characteristics of participants at the FE colleges will also be summarised by treatment arm. No statistical significance testing will be performed.

\section{Main outcomes analysis}

Recruitment rates (colleges and students) will be calculated as proportions with corresponding 95\% CIs. This will include the proportion of colleges participating in the study of the total number of colleges asked to participate, and, where available, the proportion of students who were eligible of the total number of students who were assessed for eligibility. The proportion of students tested, who obtain a positive test result and receive treatment, will be calculated at 1 and 4 months for the intervention group and at 7 months for both the intervention and the control groups, and corresponding 95\% CIs will be presented.

The time taken to recruit students will be summarised by means and standard deviations or medians and inter-quartile ranges as appropriate, for each site and overall. At intervention colleges we will summarise the time from providing a sample to treatment for students with a positive chlamydia test at 1 and 4 months with means and standard deviations or medians and inter-quartile ranges as appropriate. All confidence intervals will be two-sided and will be at the $95 \%$ level. A detailed statistical analysis plan has been developed and approved by the Trial Steering Committee [19]. 


\section{Discussion}

To our knowledge this will be the first UK cluster randomised study exploring the feasibility of rapid chlamydia tests and same-day, on-site treatment in the community. If the findings lead to a main trial which shows that TnT is acceptable, cost-effective and reduces chlamydia rates, implementing TnT in the community might improve the sexual health of many hard-to-reach young people.

\section{Trial status}

We are recruiting participants.

\section{Additional file}

Additional file 1: Standard Protocol Items: Recommendations for Interventional Trials (SPIRIT) 2013 Checklist: recommended items to address in a clinical trial protocol and related documents. (DOC $121 \mathrm{~kb}$ )

\section{Abbreviations}

FE colleges: Further education colleges; GUM: Genitourinary medicine; HPV: Human papillomavirus; ICC: Intraclass correlation coefficient; STI: Sexually transmitted infection; TnT: Test $n$ Treat

\section{Acknowledgements}

We thank Georgie Timson and Alice Bonnissent of Cepheid International for providing the rapid chlamydia/gonorrhoea tests.

\section{Funding}

This independent research is funded by the NIHR under its Research for Patient Benefit (RfPB) Programme (Grant Reference Number PB-PG-101435007). The views expressed are those of the authors and not necessarily those of the NHS, the NIHR or the Department of Health. The funding body will have no role in the design of the study, the collection, analysis or interpretation of the data, or the write-up of the manuscript.

The BMA Foundation for Medical Research TP Gunton award and the Wellcome Trust Institution Strategic Support Fund grant number 204809/Z/ 16/Z will fund the HPV and chlamydia/gonorrhoea tests in oropharyngeal samples.

\section{Availability of data and materials}

Data and materials may be obtained from SKB.

\section{Authors' contributions}

PO, FR, STS, VMD, EA, and SKB designed the study and obtained the funding. FR and RP designed the statistical analysis plan and will analyse the data with trial manager SKB. SKB and CF wrote the first draft of the study protocol to which all authors (FR, RP, VMD, EJA, WM, AB, EHE, EC, STS) then contributed. All authors read and approved the final manuscript.

\section{Ethics approval and consent to participate}

The study was approved by Bromley REC reference 15/LO/1929. All participants will provide informed written consent.

\section{Competing interests}

Pippa Oakeshott is a member of the NIHR South London Collaboration for Leadership in Applied Health Research and Care. PO, STS, EHE, and EC are members of the esti2 consortium funded under the UKCRC Translational Infection Research Initiative supported by the Medical Research Council (Grant Number G0901608) with contributions from the Biotechnology and Biological Sciences Research Council, the National Institute for Health Research.

\section{Publisher's Note}

Springer Nature remains neutral with regard to jurisdictional claims in published maps and institutional affiliations.

\section{Author details}

'Population Health Research Institute, St George's, University of London, London SW17ORE, UK. 'Department of Primary Care and Public Health Sciences, King's College London, 4th Floor, Addison House, Guy's Campus, London SE1 1UL, UK. ${ }^{3}$ Centre for Health and Social Care Research, Kingston University and St George's University of London, London SW17ORE, UK.

${ }^{4}$ Aquarius Population Health Limited, 58a Highgate High Street, London N6 5HX, UK. ${ }^{5}$ WEM Consultancy Ltd., 96 Tantallon Road, London SW12 8DH, UK. ${ }^{6}$ Infection and Immunity, St George's, University of London, London SW17ORE, UK.

Received: 26 April 2017 Accepted: 4 May 2018

Published online: 05 June 2018

\section{References}

1. Oakeshott P, Aghaizu A, Reid F, Howell-Jones R, Hay PE, Sadiq ST, et al. Frequency and risk factors for prevalent, incident, and persistent genital carcinogenic human papillomavirus infection in sexually active women: community based cohort study. BMJ. 2012;344:e4168.

2. Oakeshott P, Aghaizu A, Hay P, Reid F, Kerry S, Atherton H, et al. Is Mycoplasma genitaliium in women the 'new chlamydia'? Community-based prospective cohort study. Clin Infect Dis. 2010;51:1160-6.

3. Oakeshott P, Kerry S, Aghaizu A, Atherton H, Hay S, Taylor-Robinson D, et al. Randomised controlled trial of screening for Chlamydia trachomatis to prevent pelvic inflammatory disease: the POPI (prevention of pelvic infection) trial. Br Med J. 2010;340:1642

4. Aghaizu A, Reid F, Kerry S, Hay PE, Mallinson H, Jensen JS, et al. Frequency and risk factors for incident and redetected Chlamydia trachomatis infection in sexually active, young, multi-ethnic women: a community based cohort study. Sex Transm Infect. 2014;90:524-8.

5. National Chlamydia Coalition. Getting more young women screened for chlamydia: findings from qualitative research, vol. 3; 2011. p. 1-17.

6. Public Health England. Opportunistic chlamydia screening of young adults in England. London: Public Health England; 2014.

7. van den Broek IV, van Bergen JE, Brouwers EE, Fennema JS, Gotz HM, Hoebe CJ, et al. Effectiveness of yearly, register based screening for chlamydia in the Netherlands: controlled trial with randomised stepped wedge implementation. BMJ. 2012;345:e4316.

8. Adams EJ, Ehrlich A, Turner KM, Shah K, Macleod J, Goldenberg S, et al. Mapping patient pathways and estimating resource use for point of care versus standard testing and treatment of chlamydia and gonorrhoea in genitourinary medicine clinics in the UK. BMJ Open. 2014:4(7):e005322.

9. Turner KM, Round J, Horner P, Macleod J, Goldenberg S, Deol A, et al. An early evaluation of clinical and economic costs and benefits of implementing point of care NAAT tests for Chlamydia trachomatis and Neisseria gonorrhoea in genitourinary medicine clinics in England. Sex Transm Infect. 2014:90(2):104-11.

10. Gaydos CA, Van Der Pol B, Jett-Goheen M, Barnes M, Quinn N, Clark C, et al. Performance of the Cepheid CT/NG Xpert Rapid PCR Test for detection of Chlamydia trachomatis and Neisseria gonorrhoeae. J Clin Microbiol. 2013; 51(6):1666-72.

11. Guy RJ, Natoli L, Ward J, Causer L, Hengel B, Whiley D, et al. A randomised trial of point-of-care tests for chlamydia and gonorrhoea infections in remote Aboriginal communities: Test, Treat ANd GO- the 'TTANGO' trial protocol. BMC Infect Dis. 2013;13:485.

12. Oakeshott P, Kerry S, Atherton H, Aghaizu A, Hay S, Taylor-Robinson D, et al. Community-based trial of screening for Chlamydia trachomatis to prevent pelvic inflammatory disease: the POPI (Prevention Of Pelvic Infection) trial. Trials. 2008;9:73.

13. Normansell R, Drennan V, Oakeshott P. Exploring female FE college students' attitudes to regular sexually transmitted infection screening: a qualitative study. Health Expect. 2016;19:322-30. PMC5055273

14. Ellis J, Green R, Kerry SR, Jesuratnam G, Rajamanoharan A, Patel R, et al. Acceptability of providing self-taken vaginal samples and allowing access to NHS numbers and medical records: feasibility study in young female genitourinary medicine clinic attenders. Sex Transm Infect. 2012;88(4):300.

15. Hocking JS, Vodstrcil L, Huston WM, Timms P, Chen M, Worthington K, et al. A cohort study of Chlamydia trachomatis treatment failure in women: a study protocol. BMC Infect Dis. 2013;13(1):379. 
16. Hunjan T, Kerry SR, Hay P, Planche T, Sadiq ST, Oakeshott P. Chlamydia testing: where are we now? Recruiting high risk women to a pilot STI screening trial. Sex Trans Infect. 2013;89:556.

17. Aghaizu A, Adams EJ, Turner K, Kerry S, Hay P, Simms I, et al. What is the cost of pelvic inflammatory disease and how much could be prevented by screening for chlamydia trachomatis? Cost analysis of the Prevention of Pelvic Infection (POPI) trial. Sex Transm Infect. 2011;87(4):312-7.

18. Teare MD, Dimairo M, Shephard N, Hayman A, Whitehead A, Walters SJ. Sample size requirements to estimate key design parameters from external pilot randomised controlled trials: a simulation study. Trials. 2014;15:264.

19. Phillips R, Reid F. Test $n$ Treat statistical analysis plan. "Test $n$ Treat": a cluster randomised feasibility trial of frequent rapid testing and same day on-site treatment to reduce rates of chlamydia in further education college students. Trials. [in press] 2018.

Ready to submit your research? Choose BMC and benefit from:

- fast, convenient online submission

- thorough peer review by experienced researchers in your field

- rapid publication on acceptance

- support for research data, including large and complex data types

- gold Open Access which fosters wider collaboration and increased citations

- maximum visibility for your research: over $100 \mathrm{M}$ website views per year

At BMC, research is always in progress.

Learn more biomedcentral.com/submissions 